\title{
L'évaluation comme instrument d'effacement du sens politique : la controverse autour de l'évaluation des schools vouchers américain
}

\section{Arnaud Lacheret}

Résumé : Le chèque éducation, inventé par Milton Friedman dans les années 1960 et testé dans plusieurs Etats américains a fait l'objet d'une importante littérature outre-Atlantique et d'une campagne d'évaluation dans les années 1990-2000. Cette évaluation a donné lieu à de très nombreuses controverses dont nous soulevons ici les principales. L'effet le plus important de cette fièvre évaluatrice américaine autour du voucher a eu pour effet de dépolitiser le chèque pour en faire un outil purement technique et plus facilement exportable dans le monde entier jusqu'à sa diffusion au sein des collectivités françaises sous des formes a priori dépourvues de sens politique.

Théorisé par le Prix Nobel d'économie et père du monétarisme Milton Friedman (Friedman, 1962), le chèque éducation, ou school voucher, est conçu outre atlantique comme un dispositif libéral permettant aux parents de choisir librement l'école de leurs enfants. Les chèques ont été testés sur de nombreux terrains, et d'abord au Chili sous la dictature de Pinochet, à qui Friedman a dispensé plusieurs conseils sur sa politique économique ${ }^{1}$. Plusieurs expérimentations locales ont aussi été menées aux Etats-Unis puis le chèque éducation a été diffusé dans de nombreux Etats souvent porté par des organisations internationales.

Concrètement, le chèque éducation est une sorte de bon d'achat permettant à certaines familles (on trouve des chèques destinés à toutes les familles et d'autres pouvant viser les plus modestes par exemple) de financer les frais de scolarité d'une école privée (souvent une high school aux Etats-Unis) de façon à favoriser le libre-choix de l'établissement scolaire de leurs enfants. Il permet ainsi de contourner la logique de la carte scolaire en vigueur dans plusieurs Etats qui impose une scolarisation dans les établissements publics situés au plus près du domicile et est réputée inégalitaire puisque les ménages les plus pauvres ne peuvent financer un changement d'établissement vers une école privée. L'OCDE par exemple, le définit comme un «dispositif de financement par lequel les parents reçoivent de l'État pour chaque enfant d'âge scolaire des droits qu'ils peuvent faire valoir auprès d'une série prédéfinie

\footnotetext{
${ }^{1}$ Milton Friedman a rencontré les autorités de l'époque à plusieurs reprises et est directement l'inspirateur du chèque éducation Chilien, créé en 1980. http://www.washingtonpost.com/blogs/answer-sheet/wp/2012/10/25/ consulté le 30 août 2013
} 
d'établissements scolaires. Ceux-ci se les font ensuite rembourser par l'État. »(OCDE, 2007).

Très connoté politiquement, cet instrument d'action publique (Lascoumes et Le Galès, 2004) fait l'objet d'une littérature abondante aux Etats-Unis, et s'est développé sous plusieurs formes alternatives. On trouve ainsi par exemple des chèques logement, des chèques santé, ou des chèques alimentation qui sont très utilisés aux Etats-Unis et se sont répandus dans le monde entier jusqu'à atteindre les collectivités territoriales françaises (Lacheret, 2014).

Les analystes du chèque, au premier rang desquels on trouve Eugene Steuerle (Steuerle, 2000) ou Lester Salamon (Salamon, 2002) essaient au maximum de dépolitiser le chèque et de n'en faire qu'un outil de politiques publiques parmi d'autres dans ce qui s'apparente à une tentation de rendre le dispositif plus présentable politiquement. Cette tendance à masquer le côté libéral du chèque se retrouve jusque dans les travaux les plus récents sur le sujet comme peut en témoigner l'article de Gary Sturgess et Ivana Bodroza (Sturgess et Bodroza, 2011) pourtant publié sous l'égide du très libéral Serco Institute.

L'introduction de l'article balaie ainsi d'emblée les procès en néolibéralisme qui réduiraient le chèque à une mesure «pro-market and small government » en indiquant que le système de «vouchers » fonctionnant le mieux viendrait de Suède. Cette tentation de la dépolitisation du chèque a été portée par une vague très importante de travaux d'évaluation des différentes expériences de chèques éducation notamment aux Etats-Unis.

L'évaluation en tant qu'instrument est un moyen de techniciser l'action publique, elle a cependant un sens politique et est utilisée dans un but précis (Spenlehauer, 1998). Le présent article vise à effectuer un panorama des controverses autour de l'évaluation dont a fait l'objet le chèque éducation au sein de la communauté scientifique américaine entre 1998 et 2001. La communauté des politologues, économistes et spécialistes de sciences de l'éducation a en effet été mobilisée autour de débats riches et parfois très vifs afin de légitimer l'extension au niveau fédéral du school voucher. L'évaluation a ici joué un rôle éminemment politique et a été instrumentalisé pour atteindre cet objectif. Si le school voucher n'a pas été étendu au niveau fédéral, le caractère libéral du chèque a été gommé par ses évaluations techniques, permettant une large diffusion internationale de cet instrument.

\section{L'évaluation comme légitimation technique des choix passés}


Sans entrer dans les recherches récentes sur la notion d'évaluation, le remplacement du politique par le technique, ou plutôt la volonté de légitimer par des avis d'experts des choix politiques pouvant être perçus comme partisans est parfaitement expliquée très tôt par Jean Leca. Pour lui, l'évaluation s'apparente « à un mouvement de modernisation qui substitue au pilotage par les principes un pilotage par les conséquences » et vise la « recherche des conséquences des actions publiques, des causes qui les ont produites, des réactions complexes à celles-ci, et des moyens dont disposent les agents pour mieux les gérer »(Leca, 1993, 185).

Ce passage par la phase «évaluation » des politiques de chèque éducation aux Etats-Unis et partout ailleurs répondrait donc à une volonté de dépolitiser le chèque, de n'en faire qu'un outil que l'on peut piloter par l'évaluation, au sens de Michel Conan: "L'objectif de l'évaluation constructive est de permettre aux acteurs de réorienter leurs actions à partir d'un approfondissement des valeurs qu'ils partagent $\gg($ Conan, 1998).

La vague d'études sur l'impact et l'évaluation des chèques commandées dans les années 1990 et au début des années 2000 aurait donc pour objectif de réorienter les actions des pouvoirs publics à propos d'un outil qu'ils utilisaient depuis très longtemps (plus de 20 ans pour le chèque éducation dans la ville de Milwaukee par exemple.) Les commanditaires de ces études sont soit les autorités publiques les mettant en œuvre et souhaitant en modifier la portée, soit des think tanks libéraux (Brooking institute, Economic Policy Institute...) ou plus conservateurs (Manhattan Institute, Cato Institute...)

Si l'évaluation peut être définie comme un instrument d'action publique au sens de Pierre Lascoumes et Patrick Le Galès (Lascoumes et Le Galès, 2004, 13), elle a eu, dans ce cas précis, pour effet de dépolitiser et de légitimer un outil identifié et marqué politiquement et économiquement.

\section{L'inévitable chèque éducation}

Si la politique du chèque renvoie à de multiples pratiques et à d'évidentes origines néolibérales (Lacheret, 2014), les études d'évaluation des politiques publiques utilisant cet outil sont souvent elles-mêmes empreintes de considérations idéologiques. Il faut donc forcément tenir compte de cet éclairage souvent partisan et orienté des chercheurs avant d'entamer une recherche sur les «effets » du chèque. 
Il conviendra tout d'abord de se focaliser sur des «vouchers» ayant été testés dans un maximum de contextes, de régions et de pays. Pour cela, une étude des évaluations du «voucher fondateur » de Friedman, le chèque éducation, apparait tout à fait appropriée. Pour cela, nous avons procédé à une analyse de textes produits par des politistes, sociologues de l'éducation et surtout des économistes principalement américains auteurs d'évaluations de dispositifs de «school vouchers». Nous nous sommes concentrés majoritairement sur les textes produits au début des années 2000 qui ont correspondu avec une véritable vague d'évaluations de dispositifs locaux en privilégiant les chèques ayant donné lieu à des publications multiples et à des débats, parfois très engagés, entre scientifiques. Cette montée en puissance des évaluations des «schools vouchers » a fait l'objet d'une analyse a posteriori de l'économiste Patrick Mac Ewan (Mac Ewan, 2004) qui tente de définir une méthodologie d'évaluation de ces politiques publiques sur laquelle nous nous sommes appuyés pour mieux appréhender ce qui fut une véritable controverse outre-Atlantique.

Loin de prétendre à l'exhaustivité, et encore moins à l'exactitude puisqu'ils sont souvent teintés d'une idéologie à peine masquée, les différents rapports et articles produits sur l'évaluation ou les effets d'une politique de chèque montrent avant tout la façon dont est appréhendée et réalisée cette évaluation plus que les résultats de cette dernière qui, nous le verrons, ne permettent jamais de trancher dans un sens ou dans un autre.

1. Une méthodologie d'évaluation des effets d'un «school voucher » sur le niveau des élèves

\section{a. Des politiques différentes en fonction du ciblage de la population bénéficiant du chèque}

Dans son article intitulé «The potential impact of vouchers » qui tente de faire une synthèse des différentes méthodes d'évaluation des «schools vouchers», Patrick Mac Ewan (Mac Ewan, 2004) s'interroge sur le public se voyant attribuer les chèques. Le ciblage étant différent en fonction des cas étudiés, il distingue trois grands schémas en termes d'éligibilité à ces dispositifs : 
- D’abord, lorsque seules les familles les plus pauvres sont ciblées et bénéficient de chèques.

- Ensuite, lorsque seules les familles étant dans une zone géographique où les établissements sont peu performants peuvent se voir attribuer le chèque éducation.

- Enfin un school voucher peut être attribué à toutes les familles sans distinction (c'est le cas notamment du chèque éducation Chilien) dans une optique tout à fait conforme à l'idée initiale de Milton Friedman pour qui le choix devait être proposé à l'ensemble de la population et qui conseilla d'ailleurs en termes de politique économique le régime de Pinochet dès l'arrivée au pouvoir du dictateur².

\section{b. L'effet du chèque sur les résultats scolaires}

L'effet économique d'un chèque éducation sur le marché devrait logiquement conduire, dans les conditions d'une économie où la concurrence serait pure et parfaite, à une harmonisation du marché de l'éducation avec un secteur privé qui serait ouvert à la mixité sociale et un secteur public motivé pour progresser et s'aligner sur les standards d'excellence et de qualité du privé.

Ce raisonnement, qui est tout droit tiré des théories économiques néoclassiques, est évidemment purement théorique. C'est cette hypothèse que les chercheurs ont tenté de vérifier ou d'infirmer, se livrant à une controverse qui semble aujourd'hui s'être momentanément épuisée.

Ce raisonnement suppose aussi qu'il existerait une corrélation claire entre les ressources d'un établissement scolaire et les résultats de ses élèves. Ce débat, assez vif outre-Atlantique puisque constituant un des dogmes des contempteurs des vouchers, a notamment était alimenté par Eric Hanushek qui estime que le niveau des résultats scolaires dans un établissement est davantage dû au niveau de ressources des parents des étudiants (Hanushek, 1997).

\footnotetext{
${ }^{2}$ L'activiste Naomi Klein évoque ce rôle de conseiller dans une tribune du Guardian intitulée «Milton Friedman did not save Chile»: http://www.guardian.co.uk/commentisfree/cifamerica/2010/mar/03/chile-earthquake consulté le 30 août 2013
} 
Un établissement comptant des étudiants issus de milieux sociaux défavorisés aurait donc beau avoir toutes les ressources possibles, les résultats moyens resteraient plus bas en comparaison avec un établissement dont les étudiants viendraient de milieux aisés indépendamment des ressources propres de l'école. Le recours au privé, pour Hanushek, ne serait donc pas forcément systématiquement la meilleure des solutions dans l'enseignement.

Patrick Mac Ewan, cherchant à définir la meilleure des méthodes d'évaluation d'un chèque, s'interroge également sur les impacts réels d'un système de school voucher. Il pose ainsi trois questions essentielles auxquelles une étude d'impact d'un chèque éducation doit pouvoir être en mesure de répondre :

- Les élèves allant dans des écoles privées grâce à un chèque obtiennent-ils de meilleurs résultats que ceux restant dans le secteur public?

- Le chèque encourage-t-il le mélange des élèves (en fonction de leur statut social notamment), et cette mixité affecte-t-elle les résultats des élèves?

- Le fait de remettre des chèques rend-t-il les établissements publics plus performants et les élèves restant dans le public voient-ils leurs résultats progresser?

Pour évaluer l'effet d'une politique publique utilisant des vouchers, il est indispensable pour l'auteur que ces trois questions trouvent une réponse. Il indique toutefois que la taille de la population éligible au dispositif peut compter énormément, et que les effets seront forcément différents pour un voucher très ciblé et pour un voucher distribué à toute une population.

\section{c. Les biais des méthodes quantitatives}

La méthodologie traditionnellement retenue pour l'évaluation d'un chèque éducation est celle de «l'inférence causale» (Shadish et al, 2002). Appliquée à l'éducation, il suffira, même si de nombreux biais sont relevés, d'observer le niveau moyen d'un groupe d'étudiants passés dans le privé grâce à l'usage d'un chèque et de le comparer au niveau moyen d'un groupe d'étudiants de même niveau social restés dans le secteur public. 
Toute chose égale par ailleurs, on pourra déduire que la cause d'une éventuelle hausse du niveau du groupe étudié est directement due au passage du public au privé, ce qui pourra confirmer l'hypothèse qui constitue le fondement du chèque éducation.

Mac Ewan soulève toutefois des biais importants dans cette méthodologie et notamment le fait qu'un étudiant doté d'un chèque qui fait le choix de changer d'établissement sera sans doute plus motivé et plus attiré par la réussite qu'un étudiant s'étant vu attribuer le même chèque mais ayant choisi de rester dans son établissement. Les facteurs non observables rendent donc très compliquées les évaluations quantitatives des chèques éducation.

\section{Des progrès mesurés... et controversés...}

Malgré ces biais de telles études ont été menées, à des périodes assez similaires, sur des villes américaines et des Etats qui attribuaient des vouchers permettant à des étudiants de quitter les établissements publics dans lesquels ils étaient scolarisés afin d'intégrer des écoles privées.

\section{a. La controverse New-Yorkaise}

Ainsi, après trois années d'étude quantitative suivant la méthode définie par Mac Ewan, William Howell et Paul Peterson démontrèrent que les afro-américains de New-York ayant bénéficié du voucher et ayant choisi une école privée voyaient leurs résultats scolaires augmenter de 9,1\% par rapport à ceux ayant choisi de rester dans leur établissement public d'origine (Howell et Paterson, 2002).

Toutefois, la même étude, menée par les mêmes chercheurs, sur une population similaire sur les villes de Washington D.C et Dayton n'a démontré aucun progrès, si ce n'est pour les 10\% de meilleurs étudiants de chaque strate, sans qu'ils puissent fournir une explication. Pour des raisons que les auteurs ne parviennent pas non plus à identifier, les autres catégories ethniques étudiées (hispaniques à New-York, blancs à Dayton) ne présentaient aucun progrès scolaire mesurable malgré le fait que des vouchers leur étaient attribués et qu'ils avaient changé d'établissement.

Reprenant les données collectées par Howell et Peterson sur New-York mais en utilisant un échantillon plus important, Alan Krueger et Pei Zhu obtinrent des résultats moins probants, ne 
parvenant plus à démontrer de résultat tangible y compris pour les étudiants afro-américains (Krueger et Zhu, 2002).

Cet épisode donna lieu à de nombreux débats d'experts en statistiques et à une controverse riche puisque Howell et Peterson répondirent en 2004 à Krueger et Zhu sur un point méthodologique (Howell et Peterson, 2004), les accusant de biais dans leur définition de ce qu'était un afro-américain.

Les statisticiens David Myers et Daniel Mayer, firent également irruption dans ce débat sur ce sujet épineux aux Etats-Unis que constitue la définition sociologique d'un afro-américain (Mayer et Myers, 2003)

L'intérêt autour de ce sujet particulièrement important aux Etats-Unis, puisque mêlant à la fois politique du chèque et question raciale, fut une nouvelle fois relancé en 2004 autour d'un article intitulé «Voucher Research Controversy» (Howell et Peterson, 2004).

Cette controverse que l'on pourrait encore développer tant les références à ces travaux sont importantes montrela difficulté que rencontrent les chercheurs et les analystes pour mesurer l'impact d'une politique basée sur le voucher.

Les méthodes quantitatives sont donc systématiquement sujettes à débats et peuvent être rediscutées à l'infini au sein de la communauté scientifique ce qui ne permet pas réellement de conclure de façon définitive sur l'efficacité d'un dispositif par rapport à un autre.

Pour évaluer de façon quantitative les effets d'un programme de school vouchers, il convient plutôt de se pencher vers des systèmes hybrides comme celui, antérieur à celui de New York, ayant cours dans la ville de Milwaukee (il est d'ailleurs considéré comme le plus ancien programme de vouchers aux Etats-Unis) où, devant le grand nombre de demandes de transferts du public vers le privé, la municipalité décida d'organiser un tirage au sort aléatoire parmi les candidats à l'attribution d'un chèque.

\section{b. La «loterie » de Milwaukee}

Le biais de la motivation (autrement dit, le postulat selon lequel un candidat s'étant vu attribuer un voucher et n'ayant pas volontairement fait la démarche de quitter son établissement serait moins motivé et donc que ses résultats ne pourraient être comparés) 
pouvait ainsi être contourné et deux études menées en 1998 d'une part par Jay Greene, Paul Peterson et Jiangtao Du (Greene et al, 1998), et d'autre part par Cecilia Rouse (Rouse, 1998) conclurent séparément à une amélioration des étudiants sur certains critères (ils observèrent notamment des progrès en mathématiques).

Ces évaluations, dont on peut citer des exemples partout aux Etats-Unis tant la littérature en regorge, ont une utilité principale qui est de permettre d'orienter les choix politiques. Ainsi, dans la ville de Milwaukee, les conclusions des études de 1998 eurent pour effet d'élargir les écoles éligibles au voucher, la municipalité choisissant d'ouvrir le chèque aux écoles religieuses en se basant sur ces résultats.

Cet élargissement des écoles habilitées à recevoir des chèques eut un effet qui dépassa les frontières de l'Etat du Wisconsin. On observa notamment une extension du dispositif à Cleveland dans l'Ohio, suite à cette étude sur laquelle s'appuyèrent les autorités de la ville.

Toutefois, ces études ne donnèrent de résultats tangibles sur les notes des élèves que lorsqu'elles furent utilisées au sein d'un système où la demande trop importante de départ du public vers le privé nécessitait un tirage au sort et surtout lorsque l'échantillon étudié était suffisamment petit.

En effet, une étude menée par John Witte qui comparait les résultats d'un échantillon d'élèves de Milwaukee ayant utilisé un voucher pour se rendre dans une école privée et un échantillon aléatoire d'élèves d'écoles publiques invalida les résultats trouvés (Witte, 1998).

Les conclusions de l'évaluation du premier et probablement plus ancien véritable programme de chèques éducation à l'échelle d'une ville américaine sont donc souvent assez peu évidentes, même si les autorités n'ont retenu que celles qui allaient dans le sens d'une extension du programme puisque telle était apparemment leur volonté.

\section{c. De nouveaux biais lorsque l'échantillon s'élargit.}

Une équipe d'économistes dirigée par Joshua Angrist (Angrist et al, 2002) a réalisé une autre étude sur un échantillon beaucoup plus large d'élèves en Colombie. Ce pays a en effet lancé en 1991 un programme de chèque éducation à grande échelle, destiné aux enfants des familles les plus pauvres, appelé «Programa de Ampliacio de Cobertura de la Educacio Secundaria » (PACES). 
Le système ayant été victime de son succès, les autorités ont dû procéder à un système de tirage au sort comme à Milwaukee, mais à l'échelle du pays entier.

Se basant sur les résultats scolaires de 125000 élèves ayant bénéficié du programme, Angrist et son équipe conclurent que le taux de réussite à l'examen national équivalent au baccalauréat était supérieur de $10 \%$ entre les vainqueurs de la loterie, et les perdants trois ans après le tirage au sort. Ce résultat est néanmoins très vite modéré par le fait que la note obtenue est très faiblement supérieure et permet tout juste aux élèves de réussir leur examen.

Les auteurs, qui ont doublé cette enquête quantitative d'une étude qualitative, s'aperçoivent en outre que les perdants sont beaucoup plus démotivés puisqu'ils n'intègrent pas l'école de leur choix, ce qui pourrait expliquer l'écart déjà faible en termes de notes entre les écoles privées et publiques et constituer un biais encore plus important que celui des étudiants bénéficiaires d'un voucher et choisissant de rester au sein de leur établissement réputé de mauvaise qualité.

L'un des postulats fondamentaux du chèque éducation, repris par tous les acteurs ayant une approche idéologique du chèque en général, est que le secteur privé serait forcément meilleur que le secteur public dans quasiment tous les domaines en général et dans celui de l'éducation en particulier. Force est de constater que cette hypothèse n'a tout simplement pas été démontrée suite aux nombreuses études empiriques américaines et internationales.

$\mathrm{Au}$ contraire, les quelques études qualitatives ont prouvé que, parmi les familles les plus pauvres qui se voyaient attribuer des chèques éducation, celles qui étaient en mesure de pousser leurs enfants vers des écoles performantes et prestigieuses étaient déjà parmi les plus éduquées et cultivées (Mac Ewan, 2000).

De plus, les études économiques ont également montré que les titulaires du chèque se rendant dans des écoles privées avaient tendance à se regrouper par type ethnique (les blancs «pauvres» ont tendance à se rendre dans les écoles privées prestigieuses, elles-mêmes, composées dans leur majorité de blancs «riches ») (Figlio et Stone, 2001).

Pour David Figlio et Joe Stone, qui reprennent les conclusions des principales études monographiques sur le sujet, une politique de chèque éducation aurait même plutôt comme effet de renforcer "l'écrémage» des étudiants dans les écoles privées si elle n'était pas doublée d'une politique de réduction des inégalités, soit l'inverse de l'effet recherché. 
Les données raciales et socioéconomiques seraient donc, au vu des évaluations, des facteurs expliquant davantage la réussite ou l'échec scolaire des élèves que la qualité supposée de l'école ou son mode de gestion. Il reste désormais à examiner l'autre postulat des partisans du chèque éducation pour qui la concurrence entre les écoles serait bénéfique et permettrait aux moins bons établissements de se hisser au niveau des meilleurs.

\section{Effets de la concurrence entre écoles}

\section{a. Le chèque chilien ou le creusement d'inégalités entre écoles publiques et privées}

Le Chili, qui fut l'un des terrains de prédilection des chercheurs sur les school vouchers a également fait l'objet de nombreuses évaluations. Une des études les plus importantes basées sur le système chilien de voucher généralisée à l'ensemble du pays et à toutes les catégories sociales, a été réalisée par Chang-Tai Hsieh et Miguel Urquiola (Hsieh et Urquiola, 2006). Les conclusions suggèrent que le principal effet de ce chèque aura été de creuser les écarts entre les écoles soit l'inverse du postulat des promoteurs du chèque pour qui la mise en concurrence devrait avoir un effet bénéfique pour l'ensemble des acteurs.

Le marché des écoles privées aura évidemment été considérablement renforcé par cette aide et le secteur public de l'éducation aura connu, suite à l'application de cette mesure radicale en 1980 par l'administration de Pinochet, une baisse généralisée des résultats scolaires de ses élèves ainsi qu'une baisse du niveau socioéconomique des familles y ayant maintenu leurs enfants. Les écoles publiques mises ainsi en concurrence n'auront donc pas pu bénéficier de l'effet de la libre-concurrence pour fournir un service plus efficace, allant en contradiction complète avec les théories du chèque éducation. Ce lancement d'un chèque éducation au Chili en 1980 s'explique assez aisément, nous l'avons souligné plus haut, par la proximité idéologique en terme économique entre le dictateur chilien et le père du concept, Milton Friedman qui fut notamment l'artisan du démantèlement de la plupart des monopoles publics peu après sa prise de pouvoir de 1974 (Valdès, 1995, Garate, 2010).

Se défendant de soutenir un régime dictatorial, Friedman affirma d'ailleurs à plusieurs reprises que sa solution, ainsi que celle de l'école de Chicago, était que l'introduction du libéralisme dans l'économie chilienne avait rendu possible la transition démocratique dans les années 1990. Il fit d'ailleurs le pari que l'introduction d'une dose importante de libéralisme économique aurait les mêmes effets en Chine. 


\section{b. Le choix de l'école se ferait en fonction d'autres facteurs que son niveau scolaire}

Le creusement des inégalités est dû, selon les études menées sur cette idée du libre-choix scolaire, au fait que les parents de familles pauvres, lorsqu'on leur donne le choix, choisiront l'école de leurs enfants sans vraiment se soucier du niveau réel de cette dernière, qui est assez difficilement vérifiable, mais plutôt en évaluant le niveau socioéconomique des familles des élèves présents dans l'école convoitée.

Dennis Epple, David Figlio et Richard Romano démontrent en effet qu'il est complexe d'évaluer le niveau d'une école privée. Le niveau scolaire des enfants est en effet, selon ces auteurs, plus souvent fonction des revenus des parents qui transmettent des bases plus solides à leurs enfants indépendamment du niveau réel de l'école. Leurs conclusions, qui ne portent cependant pas sur le voucher, indiquent que des écoles privées de bon niveau qui offriront des bourses aux meilleurs élèves pauvres n'auront pour effet que d'affaiblir le secteur public sans pour autant rendre meilleurs les élèves qui auront été aidés (Epple et al, 2004).

A partir du moment où le choix de l'école privée par les parents titulaires d'un chèque se fait en fonction de la richesse supposée des familles des élèves de l'établissement, il apparait logique que cet effet de creusement des inégalités ne pourra qu'être accentué.

L'influence du chèque sur le choix de l'école et le fait que ce choix puisse être profitable ce qui est, du moins il faut le supposer, l'objectif essentiel d'une politique mettant en place un système de vouchers, est donc difficile à mesurer et donne des résultats contradictoires.

Ces difficultés, qui ont engendré une littérature très riche et alimenté de nombreuses controverses, ne permettent donc pas de démontrer que le fait de donner le choix à une famille de l'école dans laquelle son enfant va pouvoir étudier soit une solution porteuse de résultats tant au niveau scolaire individuel qu'au niveau de l'accroissement de la qualité de l'enseignement. Au mieux, si l'on suit les études les plus optimistes, le niveau de l'élève augmentera un peu, mais cela ne fera que creuser les inégalités entre les établissements et ne permettra pas une élévation générale du niveau scolaire.

\section{c. La concurrence généralisée pousse-t-elle le secteur public à progresser?}

L'autre postulat des partisans du chèque est un corolaire du libre choix : la compétition entre fournisseurs de biens et services serait, dans un univers de concurrence pure et parfaite, un 
moyen pour améliorer l'ensemble du marché. Les règles du libéralisme sont en effet censées pousser chaque concurrent à progresser pour attirer les clients. Sur cet aspect également, outre les conséquences que nous venons de souligner, l'effet du chèque éducation a été étudié.

Dans un système de voucher, comme dans la plupart des systèmes de financement des établissements scolaires, l'école publique perd des revenus en même temps qu'elle est censée perdre des élèves (c'est en fait le cas dans tous les systèmes : il n'est pas forcément illogique qu'une école qui perdrait des élèves pour n'importe quelle raison se voit amputée de postes d'enseignants par exemple, c'est le cas dans le système français, notamment pour les écoles situées dans les zones rurales dont l'actualité se fait l'écho tous les ans de fermetures de classes).

Il apparait donc, dans la théorie, parfaitement logique que l'école menacée prenne des mesures pour prévenir cette hémorragie qui deviendrait encore plus prévisible à partir du moment où l'Etat se mettrait à distribuer des chèques à ses élèves afin qu'ils puissent quitter l'établissement pour en choisir un autre.

Il n'existe quasiment pas d'études mesurant l'effet de la concurrence dans le temps sur les résultats d'élèves d'écoles publiques à une grande échelle. Toutefois une littérature abondante et particulièrement politisée a cherché à estimer l'effet de la concurrence du privé sur le public dans des systèmes scolaires plus localisés.

D'après Patrick Mac Ewan (Mac Ewan, 2000, 72), ces études ont d'abord cherché à définir ce qui pouvait constituer le marché local de l'enseignement. Christopher Jensen (Jensen, 2002, 477), dans une analyse critique, observe quant à lui que le choix de la taille du marché est parfois utilisée par certains chercheurs pour aller dans le sens de leur démonstration. Ce qui ne fonctionne pas à l'échelle d'une ville marchera peut-être à l'échelle du district ou du quartier jusqu'à ce que soit prouvé le fait que l'école publique mise en concurrence avec le privé progresse.

D'autres biais sont observés dans ce type d'études des bénéfices supposés de la concurrence sur un marché de l'éducation. D'abord, au sein de ce marché, les données sur le recrutement des écoles privées sont étudiées en même temps que les résultats scolaires des écoles publiques sur le même marché, sans qu'il soit supposé que l'un soit la cause de l'autre.

Ainsi, le fait que les meilleurs étudiants du privé partent dans le public n'est pas considéré comme un facteur à prendre en compte dans l'évolution - négative - des résultats des élèves. 
L'autre biais relevé est le fait que la localisation des écoles privées n'est pas prise en compte alors qu'en toute logique, il peut arriver qu'une école privée ne s'installe dans un quartier que parce que la population est plutôt riche et composée de CSP supérieures. Elle ne fera donc pas réellement concurrence à une école publique située dans un quartier géographiquement proche, mais qui ne sera pas vraiment réservée à la même «clientèle ». Thomas Dee, analysant ces biais en 1998 (Dee, 1998, 419), constatera que leur révélation n'empêchera pas les décideurs politiques de favoriser les politiques de chèques, car ils partent du principe qu'une concurrence entre système éducatif public et privé permettrait d'améliorer le premier cité. La littérature n'aura cependant de cesse d'essayer d'éviter ces biais.

L'une des tentatives intéressantes de contournement de ces biais a visé à isoler le marché de l'éducation en fonction de la population d'origine et de son origine religieuse (catholique en l'occurrence) dans un secteur donné. Une étude en 2005 très controversée conclue ainsi que le fait d'étudier dans l'enseignement catholique permet non seulement d'accroitre le niveau moyen des élèves qui en bénéficie par l'intermédiaire d'un système de chèque, mais contribue aussi à accroitre le niveau des écoles publiques au sein d'un même marché éducatif (Altonji et al, 2005, 151).

L'étude la plus complète sur les effets de la concurrence, a été menée par Clive Belfield et Henry Levin (Belfield et Levin, 2002, 279). Se basant sur 41 études empiriques déjà menées sur ce sujet et analysant les résultats tant en termes de quantité que de qualité des professeurs, de notes aux différents examens, du budget alloué, ils conclurent qu'il existait certes un lien entre la compétition entre établissements et la hausse du niveau des étudiants, ainsi que la hausse du niveau de tous les compétiteurs, mais que celle-ci était très faible.

Leur phrase de conclusion, assez philosophe, est que «nombreux sont les facteurs permettant d'améliorer le niveau scolaire ». Ils poursuivront d'ailleurs leur étude en dirigeant cinq ans plus tard un ouvrage qui aura pour ambition d'orienter la politique américaine d'enseignement primaire et secondaire (Belfield et Levin, 2007) en listant les améliorations à apporter. La concurrence ne figurera que très peu dans ce livre, tout juste pour en relativiser les effets.

\section{d. La controverse « Jay Greene » et le programme A + de Floride}

Malgré les nombreux biais et approximations relevées, les observations dans plusieurs Etats américains ont bien montré une certaine amélioration des résultats académiques des écoles publiques mal classées dont les élèves pouvaient se voir attribuer des vouchers. Jay Greene 
(Greene, 2001), dans son étude du programme « $A+»$ de vouchers en Floride, conclut que l'augmentation des résultats moyens aux examens des écoles les moins bien classées est deux fois supérieure aux écoles classées dans la moyenne (et dont les élèves sont exclus du programme de voucher). Il met cet effet sur le compte de la menace du voucher. La crainte de voir leurs budgets s'effondrer et au final de devoir fermer leurs écoles pousserait les directeurs à être plus sélectifs et à se séparer de leurs enseignants les moins performants.

La critique de cette analyse, comme souvent lorsqu'une étude tend à montrer qu'une politique basée sur un chèque éducation fonctionne, sera quasi immédiate puisque Gregory Camilli et Katrina Bulkley publièrent une critique à la suite de cet article basée sur les effets pervers de la peur du voucher et sur des erreurs de calcul des échantillons (Camilli et Bulkley, 2001). La principale critique formulée est que ce n'est pas la qualité de l'enseignement qui progresse, mais que les chefs d'établissements, en étant plus sélectifs et en éloignant les plus mauvais élèves pour faire augmenter leurs moyennes et ne plus être dans la catégorie d'établissements éligibles aux programmes de vouchers ne font que déplacer le problème, les moins bons éléments étant scolarisés dans d'autres établissements publics qui à leur tour deviennent la cible du dispositif.

Le débat sur ce dispositif de Floride fut âprement commenté et défendu puisque Jay Greene publia dans la foulée une réponse à la critique sur le site de la Harvard Kennedy School ${ }^{3}$. Jay Greene et son évaluation jugée beaucoup trop favorable du système de Floride furent également critiqués non pas du point de vue des résultats concrets, mais du point de vue de la cause de la hausse de niveau dans le secteur public.

\section{e. Des causes alternatives aux vouchers et à la concurrence pour expliquer les hausses de résultats dans l'enseignement public}

Doug Harris (Harris, 2008) essaya de démontrer que ce n'était pas la concurrence du privé par le biais du chèque éducation qui avait permis aux écoles publiques les plus faibles de Floride de voir leur niveau s'élever mais plutôt le fait que l'Etat de Floride donnait des notations aux établissements. Ces notations allaient de $\mathrm{A}$ à $\mathrm{F}$ (des «meilleures » écoles aux moins bonnes) et les élèves des 158 écoles ayant été classées dans la catégorie $\mathrm{F}$ pouvaient bénéficier de chèques pour partir dans un autre établissement sous certaines conditions.

\footnotetext{
${ }^{3}$ http://www.hks.harvard.edu/pepg/PDF/Papers/Bulkley.PDF
} 
Harris conclut que c'était davantage le fait de mettre une notation très basse à une école qui pouvait pousser celle-ci à sortir de cette situation embarrassante vis-à-vis du grand public et des médias plutôt que le fait d'attribuer des vouchers à ses élèves.

D'autres facteurs peuvent donc expliquer que la concurrence entre secteur public et secteur privé ait comme effet secondaire une hausse du niveau scolaire des étudiants dans les établissements publics. Ainsi, Helen Ladd et Elizabeth Glennie ont effectué la même étude que Jay Greene à partir de données récoltées en Caroline du Nord (Ladd et Glennie, 2001). La même expérience a également été réalisée à partir des données de l'Etat du Texas par Amanda Brownson (Brownson, 2001).

Ces Etat attribuent également des notes aux établissements sans attribuer de chèque aux élèves des écoles les moins bien classées. Or, les résultats sont les mêmes qu'en Floride : les résultats scolaires des élèves des écoles les moins bien notées progressent plus vite que ceux des écoles étant classées dans la moyenne. Les conclusions de Jay Greene sont très discutées. L'explication selon laquelle la menace de l'attribution du voucher pousserait les établissements à sortir de la zone dangereuse ne serait pas le véritable facteur explicatif des progrès assez difficilement explicables des écoles les moins bien notées.

\section{f. Un débat qui culmine en 2001, année électorale.}

L'année 2001 n'apparait pas par hasard dans l'explosion, au sein de la littérature scientifique américaine, des études sur les programmes de vouchers. Ainsi, dans l'introduction de son étude sur la Floride, Jay Greene indique de façon assez opportune et sans doute politiquement intéressée que «Les résultats sont particulièrement intéressants à cause des similarités entre le programme $A+$ de Floride et l'initiative sur l'éducation proposées par le Président George W. Bush » (Greene, 2001, 2)

Dans l'introduction de l'ouvrage collectif qu'il dirige et où sont présentées les études allant à l'encontre des conclusions de Jay Greene, Martin Carnoy précise également les enjeux de ces recherches.

Après une introduction sur les chèques éducation utilisés localement, il donne à son ouvrage une connotation nationale : "[mais] avec la candidature de George Bush et son élection à la présidence, ils sont devenus un enjeu national. Dans le même temps, les partisans du voucher [Jay Greene est clairement visé] ont produit de nouvelles études concluant que l'utilisation des vouchers par les étudiants augmentait leurs résultats académiques et que la menace de 
l'utilisation des vouchers poussait à augmenter les performances des étudiants dans les écoles publiques » (Carnoy, 2001, 6)

L'explosion des publications sur le sujet de l'évaluation et de l'impact des politiques de chèque éducation aux Etats-Unis s'explique donc aisément, tout comme les nombreuses controverses autour de la moindre étude d'impact sur le sujet.

Les considérations de politique extérieure, puis la crise économique, n'ont toutefois pas permis au Président Bush de mettre au point son «initiative education » qui était prévue dès son premier mandat. Tout juste l'administration mit-elle au point le plan «No Child Left Behind », qui fut déposée et soutenue par des sénateurs et des représentants Républicains et Démocrates (Jorgensen et Hoffmann, 2003). Cette loi fédérale est d'ailleurs souvent citée comme un exemple de législation obtenue grâce à un consensus entre les deux camps politiques américains.

Pour retrouver l'idée de chèques dans une politique fédérale américaine, il convient de remonter à une proposition présidentielle de 2007 où la secrétaire à l'éducation de l'administration Bush a tenté d'imposer au Congrès l'introduction de vouchers pour les enfants les moins performants lors des tests d'évaluation du programme «No Child Left Behind».

Les débats rapportés par le Washington Post montrent clairement une ligne de fracture entre les démocrates ayant apporté leur soutien au plan de 2001 et l'administration Bush. Ainsi, le Sénateur Démocrate Edward Kennedy indique lors des débats que «des propositions idéologiques comme les chèques éducation et les attaques sur les négociations collectives ne permettront pas d'aller vers une relance de ce programme dont les buts sont partagés et bipartisans $\gg($ Paley, 2007).

Jay Greene n'arrêtera pas pour autant de publier des études et essais assurant la promotion du choix de l'école par le biais du chèque éducation. Il attribue leur échec au niveau fédéral aux syndicats d'enseignants et à une «alliance de politiciens retranchés » dans un ouvrage au titre évocateur «Why America Needs School Choice? »(Greene, 2011)

L'évaluation du chèque éducation est donc quasiment impossible à mener à bien. Le processus d'évaluation, plus que l'objet en lui-même, fait toujours aux Etats-Unis, l'objet 
d'une controverse particulièrement vive. Le principal effet de cette vague d'évaluation et de débats sans précédents fut, nous l'avons vu, de dépolitiser au maximum la notion de school voucher et d'une façon plus large, de voucher en général.

C'est cette technicisation et cette perte de sens politique qui a pu participer à la diffusion de cet outil dans le monde entier (le système suédois pour ne citer que cet exemple est très proche du chèque éducation imaginé par plusieurs Etats américains.)

Non seulement le chèque s'est diffusé géographiquement, mais il s'est diversifié. Cet outil, conçu au départ pour choisir une école, puis dont l'utilisation s'est étendu à l'ensemble des interventions sociales s'est diffusé au sein des collectivités locales françaises dans un silence idéologique assourdissant, notamment au sein des Conseils Régionaux et Généraux : 21 régions métropolitaines utilisent ce mode de paiement à destination des lycéens notamment pour faciliter l'accès aux sports, à la culture ou plus généralement pour l'acquisition des manuels scolaires, plus d'une trentaine de départements versent tout ou partie des aides sociales obligatoires par le biais de Chèques emploi services universels ou de chèques d'accompagnement personnalisés.

Cette diffusion aurait sans doute été beaucoup plus complexe si l'objet avait été présenté comme une innovation néolibérale anglo-saxonne comme cela a été le cas lors de la première expérience de chèque culture destiné aux lycéens lancée en 1994 par le Conseil régional Rhône-Alpes. Plusieurs acteurs de ce dispositif interrogés sur la genèse de ce qui était alors présenté comme une innovation affirment d'ailleurs s'être inspirés du chèque éducation ${ }^{4}$.

Le processus de technicisation et de dépolitisation démarré par la série d'évaluations dont les échos ont évidemment été repris par les différents médiateurs internationaux a sans doute joué un rôle dans l'apparente facilité avec laquelle ces dispositifs se sont implantés dans les collectivités territoriales françaises.

\section{Arnaud Lacheret est Docteur en science politique, chercheur associé au Laboratoire Pacte (UMR 5194) de Sciences Po Grenoble et à Idrac research.}

\footnotetext{
${ }^{4}$ Entretiens avec Charles Millon ancien Président du Conseil régional (1988-1999) Rhône-Alpes le 3 juin 1999 et $1^{\text {er }}$ octobre 2011 et avec Amaury Nardone ancien Président de la Commission « culture » (1998-1999) au Conseil régional Rhône-Alpes le $1^{\text {er }}$ mars 1999 et le 10 février 2010 (Lacheret, 2014).
} 


\section{Références bibliographiques}

Altonji, Joseph, Elder, Todd et Taber, Christopher (2005), « Selection on Observed and Unobserved Variables: Assessing the Effectiveness of Catholic Schools », Journal of Political Economy, n¹13, p. 151-184

Angrist, Joshua, Bettinger, Eric, Bloom, Erik, King, Elizabeth et Kremer, Michael, « Vouchers for private schooling in Colombia: Evidence from a Randomized Natural Experiment », American Economic Review, n92, 2002, p. 1535-1558

Belfield, Clive et Levin, Henry (2002), «The effects of competition between schools on educational outcomes: A review for the United States », Review of Educational Research, vol 72, p. 279-341

Belfield, Clive et Levin, Henry (dir.) (2007), The Price We Pay: Economic and Social Consequences of Inadequate Education, Brookings Institution

Camilli, Gregory et Bulkley, Katrina (2001), «Critique of "An Evaluation of the Florida APlus Accountability Program" », Education policy analysis archives, vol. 9

Carnoy, Martin (dir.) (2008), School vouchers, examining the evidence, Economic policy institute

Conan, Michel (1998), L'évaluation constructive. Théorie, principes et éléments de méthode, Editions de L'Aube

Dee, Thomas (1998), « Competition and the quality of public schools », Economics of Education Review, ${ }^{\circ} 17$, p. 419-427

Epple, Dennis, Figlio, David et Romano, Richard (2004), «Competition between private and public schools: Testing stratification and pricing predictions », Journal of Public Economics, n ${ }^{\circ} 8$, p. $1215-1245$

Figlio, David et Stone, Joe (2001), « Can public policy affect private school cream skimming? », Journal of Urban Economics, n49, p. 240-266

Figlio, David et Page, Marianne (2002), « Can School Choice and School Accountability Coexist Successfully? » In Hoxby, Caroline (dir.), The Economics of School Choice, University of Chicago Press, p. 49-68

Friedman, Milton (1962), Capitalism and freedom, University of Chicago Press 
Garate, Emmanuel (2010), La «Révolution économique » au Chili. A la recherche de l'utopie néo-conservatrice (1973-2003), Thèse de Doctorat en Histoire, EHESS

Greene, Jay, Peterson, Paul et Du, Jiangtao (1998), « School choice in Milwaukee: A randomized experiment » in Peterson, Paul et Hassel, Bryan (eds.), Learning from school choice, Brookings Institute, p 335-356

Greene, Jay (2001), An evaluation of the Florida A-Plus accountability and school choice program. New York, Center for Civic Innovation, Manhattan Institute

Greene, Jay (2011), Why America Needs School Choice?, Encounter Broadside

Hanushek, Eric (1997), « Assessing the effect of school resources on student performance: An Update ». Educational Evaluation and Policy Analysis, n¹9, p. 141-164

Harris Doug (2008) «What caused the effects of the Florida A+ program : ratings or vouchers? » in Carnoy Martin (dir.), School vouchers, examining the evidence, Economic policy institute

Hsieh, Chang-Tai et Urquiola, Miguel (2006), «The effects of generalized school choice on achievement and stratification: Evidence from Chile's voucher program », Journal of Public Economics, ${ }^{\circ} 90$, p. 1477-1503

Jensen, Christopher (2002) « The role of aggregation in estimating the effects of private school competition on student achievement », Journal of Urban Economics, n`52, p. 477-500

Krieger, Alan et Zhu, Pei (2002), Another look at the New York City school voucher experiment, National Bureau of Economic Research

Jorgensen, Margaret et Hoffmann, Jenny (2003), History of the No Child Left Behind Act of 2001, Pearson Education

Lacheret, Arnaud (2014), L'aide sociale par le chèque, genèse et mise en cuvre des dispositifs sociaux et culturels ciblés des collectivités territoriales, Thèse de doctorat de science politique, Université de Grenoble

Ladd, Helen et Glennie, Elizabeth (2008), « A Replication of Jay Greenes's voucher effect study using North Carolina data » in CARNOY Martin (dir.), School vouchers, examining the evidence, Economic policy institute

Lascoumes, Pierre et Le Galès, Patrick (dir.) (2004), Gouverner par les instruments, Paris, Presses de la Fondation nationale des sciences politiques

Leca, Jean (1993), « Sur le rôle de la connaissance dans la modernisation et le statut de l'évaluation », Revue française d'administration publique, n66, p. 185-196

Mac Ewan, Patrick (2000), «The potential impact of large-scale voucher programs», Review of Educational Research, $\mathrm{n}^{\circ} 70$, p. 103-149 
Mac Ewan, Patrick (2004), «The Potential Impact of Vouchers », Peabody Journal of Education, ${ }^{\circ} 79$, p. $57-80$

Myers, David et Mayer, Paul (2003), « Comments on another look at the New York City Voucher Experiment », Washington DC Mathematics Policy Research

OCDE (2003), V. Davantage d'équité et de qualité dans l'investissement en capital humain, Etudes économiques de l'OCDE, n²17, p. 111-135

Paley, Amit (2007), « Bush Proposes Adding Private School Vouchers to 'No Child' Law », Washington Post, 25 janvier

Peterson, Paul et Howell, William (2004), «Efficiency, Bias and Classification Schemes - A response to Alan Krueger and Pei Zhu », American behavioral scientist, n47, p. 699-717

Peterson, Paul et Howell, William (2004), « Voucher Research Controversy - New look at the New York City evaluation », Education next, vol. 4, n²

Rouse, Cecilia (1998), «Private school vouchers and student achievement: An evaluation of the Milwaukee parental choice program », Quarterly Journal of Economics, n ${ }^{\circ} 113$, p. 553602

Salamon, Lester (dir.) (2002), The Tools of Government : A Guide to the New Governance, Oxford University Press

Shadish, William, Cook, Thomas et Campbell, Donald (2002), Experimental and quasiexperimental designs for generalized causal inference, Houghton Mifflin Company

Spenlehauer, Vincent (1998), L'évaluation, avatar de la planification, Thèse de Doctorat de science politique de l'université Pierre Mendès-France, Grenoble

Steuerle, Eugene (dir.) (2000), Vouchers and the provision of public services, Brookings Institute

Sturgess, Gary et Bodroza, Ivana (2011), Redeeming vouchers in Public Services, Serco Institute

Valdes, Juan Gabriel (1995), Pinochet's economists : the Chicago school in Chile, Cambridge University Press

Witte, John (2001), The market approach to education : an analysis of America's first voucher program, Princeton University Press

Witte, John (1998), « The Milwaukee voucher experiment », Educational Evaluation and Policy Analysis, $\mathrm{n}^{\circ} 20$, p. 229-251 